\title{
Coherent potential theory for interacting bands: Phonons and excitons in substitutionally disordered molecular crystals*
}

\author{
Hwei-Kwan Hong† and Raoul Kopelman \\ Department of Chemistry, University of Michigan, Ann Arbor, Michigan 48104
}

(Received 19 July 1972)

\begin{abstract}
A coherent potential approximation (CPA) theory for disordered molecular solids with interacting bands is reported here. This theory has a wide range of applications. Various examples of interacting bands can be cited, such as electronic states coupled via vibronic or spin-orbit couplings, vibrational states with degeneracies in the gas phase or coupled by Fermi resonance, triplet magnetic sublevels coupled via exciton interactions, and phonons in general. The theory is developed using the self-consistent condition with a single-site and single-band approximation. In particular, two approaches are adopted. In the first approach, a self-energy is assigned for each subband. In the second approach, a common self-energy is assumed for all the subbands. The two different approaches require different inputs to the theory. In one case, the entire dispersion relations of the pure system are called for; in the other, only the partial densityof-states functions for each degree of freedom are needed. It is also shown that in the limit of infinite dilution, the formalism reduces to the proper single-impurity levels within the single-band approximation.
\end{abstract}

\section{INTRODUCTION}

Recently, there is a great interest in phonons and excitons in disordered systems. Various techniques have been developed to deal with such systems. ${ }^{1}$ A particularly fruitful approach based on propagators and Feynman diagrams was first introduced by $\operatorname{Lax}^{2}$ and developed in an elegant manner by Yonezawa and Matsubara. ${ }^{3}$ An approximation known as the Coherent Potential Approximation (CPA) was also introduced subsequently by Soven, ${ }^{4}$ Taylor, ${ }^{5}$ Onodera, and Toyozawa. ${ }^{6}$ Applications of the CPA to the Frenkel exciton bands came immediately after in the works of Hong and Robinson, ${ }^{7}$ Dubovskii and Konobeev, ${ }^{8}$ and Hoshen and Jortner. ${ }^{9}$ All of these works, however, are based on the assumption that the particular band treated is an isolated exciton band. This model would not be applicable to some interesting physical systems where several interacting bands are involved.

An interesting case is that of phonons in disordered molecular crystals. It is well-known that, if the lattice dynamics of a molecular crystal is treated in a rigidmolecule approximation, the translational and rotational degrees of freedom are generally coupled. ${ }^{10}$ Furthermore, rotational motions often involve all three rotational degrees of freedom. Therefore, it is necessary, in general to deal with several interacting bands simultaneously. As a consequence, the corresponding disordered systems, with impurities having different masses and moments of inertia, would be characterized not by one, but by several "trap depths". The Coherent Potential theory for such systems has to be developed.

Various other systems of interest can also be cited. For example, in the intramolecular vibrational regions the existence of either Fermi resonance ${ }^{11}$ or crystal site splitting of gas-phase degenerate bands ${ }^{12}$ necessitates a theory of disordered systems with interacting bands. For the electronic transitions, the same complication exists in cases where two or more electronic states are coupled via spin-orbital couplings (such as a singlet and a triplet) or vibronic couplings (such as a singlet and a singlet). The interactions between these bands cannot be ignored when bands are close to each other. Another important application involves triplet impurity states. Our present understanding of the triplet state comes largely from the paramagnetic resonance of the metastable phosphorescent species in the mixed crystals. ${ }^{13}$ Since the zero field splittings are generally comparable with the exciton interactions due to electron exchange integrals, ${ }^{14}$ it is imperative to treat the magnetic sublevels in pure or mixed crystals as interacting bands. Previous work ${ }^{15}$ on the triplet impurity states ignored the zero field splittings of magnetic sublevels. Such an approch would be erroneous if shallow traps are involved. Since it is a commonplace occurrence to have a congested spectral region in molecular crystals, it is certainly worthwhile to investigate the behavior of disordered systems under this circumstance.

In this paper, we limit our discussion to the phonons and excitons of simple disordered systems of heavily doped isotopic mixed crystals. We follow the customary assumptions that (1) the impurities (or guests) are situated at substitutional sites, and (2) the impurityimpurity (guest-guest) or impurity-host (guesthost) interactions are the same as the host-host interactions. The single-site approximation of Velicky et $a .^{16}$ will be adopted in developing the CPA. We discuss how the investigation of disordered systems can be utilized to study the interactions in the ordered systems. Finally, we briefly touch on pertinent features of the Raman spectra of naphthalene and benzene lattice modes in the light of our theoretical results.

\section{THE COHERENT POTENTIAL APPROXIMA- TION FOR DISORDERED SYSTEMS}

\section{A. Single-Site Approximation}

We consider here only binary systems of varying compositions. The problem can be set up by considering 
first the Green's function corresponding to a particular distribution of guests and hosts. The Green's function can be written as

$$
G(Z)=(Z-H)^{-1},
$$

where $Z=E+i \delta$, and $\delta$ is a small quantity. $H$ is, of course, the mixed crystal Hamiltonian for a particular configuration. The macroscopic properties of the disordered systems are determined by the average Green's function. The averaging must be carried over all possible guest-host configurations, subject only to the constraint that the guest concentration is a fixed quantity. Thus, we have

$$
\langle G(Z)\rangle=\left\langle(Z-H)^{-1}\right\rangle=\left(Z-H_{\text {eff }}\right)^{-1} .
$$

Equation (2.2) actually defines $H_{\text {eff }}$. Notice that $\boldsymbol{H}_{\text {eff }}$ has the full symmetry of the crystal, ${ }^{17}$ but it is nonHermitian and energy dependent. ${ }^{16}$ In general, an approximate Hamiltonian to the exact effective Hamiltonian $H_{\text {eff }}$ will be called $K(Z) . K(Z)$ is to be determined in the CPA approach by imposing both the single-site approximation ${ }^{16}$ and self-consistency. The corresponding Green's function which is an approximation to the average Green's function is simply

$$
R(Z) \equiv(Z-K)^{-1}
$$

$R(Z)$ and $\langle G(Z)\rangle$ are related by the Dyson ${ }^{16}$ equation:

$$
\langle G\rangle=R+R\left(H_{\text {eff }}-K\right)\langle G\rangle .
$$

It is convenient at this point to introduce the $T$ matrix ${ }^{18}$ of multiple-scattering theory,

$$
G=R+R T R,
$$

which immediately leads to

$$
\langle G\rangle=R+R\langle T\rangle R .
$$

From Eqs. (2.4) and (2.6), the relation between $H_{\text {eff }}$ and $K$ can be obtained;

$$
H_{\text {eff }}-K=\langle T\rangle(1+R\langle T\rangle)^{-1} .
$$

It is important to note here that the preceding development can be used in three different ways. If one is interested in seeking the true solution, one can evaluate $T$ exactly using one's trial solution $K$ through Eq. (2.5). The correctness of this trial solution can be confirmed through Eq. (2.7) if $\langle T\rangle=0$. Actually one can even use $\langle T\rangle=0$ as the necessary and sufficient condition that $H_{\text {eff }}=K$, and solve for $K$ because $T=T(K)$. This is not very useful in practice due to the complexity of the exact solution. Alternatively, if one is interested in the deviation of the approximate solution $K$ from the exact $H_{\text {eff }}, \mathrm{Eq}$. (2.5) would determine $T$ and again through Eq. (2.7) such a deviation can be computed. A third and more useful but subtle application of the development is to obtain a self-consistent approximation to the true solution. Generally, an approximate effective Hamiltonian $K$ is assumed and so is an approximate $T$,
$\bar{T}$. This would lead to an approximate $G, \bar{G}$ and hence an approximate $\langle G\rangle,\langle G\rangle$. One would, then, complete the cycle (i.e., imposing self-consistency) by demanding $\langle\bar{T}\rangle=0$. Equation (2.7) would guarantee that approximate $H_{\text {eff }}, \bar{H}_{\text {eff }}$ equals $K$ and $\langle\bar{G}\rangle=R$. This is the approach adopted by various workers ${ }^{4,16}$ to determine the CPA self-energy. The logic involved will be more transparent as we proceed to set up the CPA theory in the following discussion.

It is frequently possible to express the random scattering potential in terms of a sum of scattering potentials due to individual sites, i.e.,

$$
H-K=\sum_{n} V_{n}
$$

In the case of phonons or excitons in molecular crystals, since each site has several degrees of freedom, a second summation over all degrees of freedom is necessary. The single configuration Green's function is related to the approximate average Green's function through the appropriate Dyson equation:

$$
G=R+R(H-K) G \text {. }
$$

Equations (2.5) and (2.9) yield

$$
\begin{gathered}
T=(H-K)(1+R T), \\
T=\sum_{n} V_{n}(1+R T),
\end{gathered}
$$

We now introduce the single site approximation in a slightly different context than was previously introduced by Velicky et al. ${ }^{16}$ First we assume that $T$ can be written as a sum of site operators,

$$
T \equiv \sum_{n} Q_{n}
$$

Equations (2.11) and (2.12) now relate $Q_{n}$ to $V_{n}$. In other words,

$$
\sum_{n} Q_{n}=\sum_{n}\left[V_{n}\left(1+R \sum_{n} Q_{n}\right)\right] .
$$

Alternatively, one can write, for individual $Q_{n}$,

$Q_{n}=\left(1-V_{n} R\right)^{-1} V_{n}+\left(1-V_{n} R\right)^{-1} V_{n} R \sum_{m \neq n} Q_{m}$.

Our single-site approximation will be introduced here to decouple $Q_{n}$ from $Q_{m}$. We will approximate $Q_{n}$ by $\bar{Q}_{n}$, which is defined as

$$
\bar{Q}_{n}=\left(1-V_{n} R\right)^{-1} V_{n}
$$

Or, on averaging,

$$
\left\langle\bar{Q}_{n}\right\rangle=\left\langle\left(1-V_{n} R\right)^{-1} V_{n}\right\rangle .
$$

Under this approximation, the approximate average $T$ matrix takes an especially simple form:

$$
\langle\bar{T}\rangle=\sum_{n}\left\langle\tilde{Q}_{n}\right\rangle
$$

An equation similar to Eq. (2.7) can then be displayed 
as

$$
\bar{H}_{\text {off }}-K=\left(\sum_{n}\left\langle\bar{Q}_{n}\right\rangle\right)\left[1+R\left(\sum_{n}\left\langle\bar{Q}_{n}\right\rangle\right)\right]^{-1} .
$$

Self-consistency is achieved if we now impose the condition

that is

$$
\left\langle\tilde{Q}_{n}\right\rangle=0 ;
$$

$$
\left\langle\left(1-V_{n} R\right)^{-1} V_{n}\right\rangle=0 .
$$

It is clear from previous developments that

and

$$
\bar{H}_{\text {eff }}=K
$$

$$
\langle\bar{G}\rangle=R \text {. }
$$

Equation (2.19) is identical to the one previously obtained by Velicky et al. ${ }^{16}$; however, Eqs. (2.16) to (2.18) are not. We have obtained the self-consistent single-site approximation $K$ through Eq. (2.19) in a simpler and more straightforward manner than Velicky's method. In comparing with Velicky's method, we should also point out that $\left\langle\bar{Q}_{n}\right\rangle$ is formally defined in a different way here. Velicky $e t a l .^{16}$ used the following definition:

$$
\left\langle\bar{Q}_{n}\right\rangle=\left\langle\left(1-V_{n} R\right)^{-1} V_{n}\right\rangle\left(1+R \sum_{m \neq n}\left\langle\bar{Q}_{m}\right\rangle\right) .
$$

However, since the self-consistency requires

$$
\left\langle\left(1-V_{n} R\right)^{-1} V_{n}\right\rangle=0,
$$

it is clear that $\left\langle\bar{Q}_{m}\right\rangle=0$. Therefore Eqs. (2.21) and (2.16) really involve the same physical approximation, namely the neglect of all statistical correlations between site $n$ and all other sites $m$ (this leads to the smearing out of the cluster states). The validity of such an approximation has been discussed in the work of Velicky et $a l^{16}$ and will not be repeated here.

\section{B. Single-Site and Single-Band Approximation for Interacting Bands}

We can now extend our previous treatment to the case of interacting bands. We assume that there are several subbands interacting with each other. Each subband (or each degree of freedom in the phonon case) is denoted by index $f$. The fluctuating potential for our systems is still given by

$$
H-K=\sum_{n} V_{n}
$$

However, at this point a careful examination of the nature of $V_{n}$ is necessary. We will begin with $H$. As we mentioned before, for the systems studied here, the perturbations attributable to the introduction of impurities are assumed to be localized with respect to both site and subband indices (see also Secs. III and IV); i.e.,

and

$$
H=H^{0}+\Delta
$$

$$
\begin{aligned}
\Delta & =\sum_{n} \sum_{n^{\prime}} \sum_{f} \sum_{f^{\prime}}|n, f\rangle \Delta_{n f}\langle n, f| \delta_{n n^{\prime},} \delta_{f f^{\prime}} \\
& =\sum_{n} \sum_{f}|n, f\rangle \Delta_{n f}\langle n, f| .
\end{aligned}
$$

where $B^{0}$ is the pure crystal Hamiltonian and $\Delta_{n f}=0$, if the $n$th site is occupied by the host and $\Delta_{n f}=\Delta_{f} \neq 0$, if it is occupied by the guest. In the exciton case, this assumption simply states that the presence of impurities does not introduce a change in the exciton interactions. In the phonon case, we assume that the force constants in mixed crystals are the same as those in the pure crystal (i.e., impurities are simple mass defects). This is the assumption of substitutional disorder and is appropriate for isotopic mixed crystals discussed here.

By the same token, the approximate effective Hamiltonian $K$ can be expressed (in the framework of CPA) as

$$
K=H^{0}+\sum_{n} \sigma_{n},
$$

where $\sum_{n} \sigma_{n}$ is the self-energy. It is obvious that $\sigma_{n}$ is nondiagonal if one uses the model in Sec. II.A. To see this, one should notice that for multiple bands Eq. (2.19) is really an $F \times F$ matrix equation, where $F$ is the total number of interacting bands. As a matter of fact, if we substitute $V_{n}=\sum_{f}|n, f\rangle \Delta_{n}\left\langle\langle n, f|-\sigma_{n}\right.$ into Eq. (2.19), we obtain $F \times F$ simultaneous equations to determine $F \times F$ matrix elements of $\sigma_{n}$. Therefore, we have to assume that $\sigma_{n}$ is an $F \times F$ matrix to assure selfconsistency. Physically speaking, one can see that although $\Delta$ does not couple different subbands, $H^{0}$ does. The net effect is that $\sigma_{n}$ is nondiagonal. This particular model is very difficult to work with. Let's consider phonons in benzene mixed crystals. There are six degrees of freedom corresponding to three rotations and three translations. Although each degree of freedom has a well-defined perturbation strength, the present formalism would call for the solution of 36 simultaneous equations in order to calculate the self-energy. Because of the complexity involved, it appears to be desirable to introduce an even lower approximation, which we call the single-site and single-band approximation. First, we notice that the form of $\Delta$ in Eq. (2.23b) is already appropriate for the single-site and single-band approximation. The only further approximation we have to make is on $\sigma_{n}$. We assume, in the single-site and singleband approximation, that $\sigma_{n}$ is diagonal in the localized representation such that a $V_{n f}$ can be defined. Under this assumption,

$$
V_{n}=\sum_{f} V_{n f} \equiv \sum_{f}\left(|n, f\rangle \Delta_{n j}\langle n, f|-\sigma_{n f}\right),
$$

which is possible only if one assumes

$$
\sigma_{n}=\sum_{f} \sigma_{n f} .
$$

In the following paragraphs, we will demonstrate how $\sigma_{n f}$ can be determined self-consistently. Notice that if one calculates $\sigma_{n}$ from Eq. (2.19) and throws out the off-diagonal terms, the resulting self-energy would not be the same as the one obtained through self-consistency. Recently Sen and Cohen ${ }^{19}$ have done some model calculations based on Eq. (2.19). They assume only two interacting bands and a simple density-of- 
states function. Similar calculations based on our model will be done to compare the two different approaches.

We now define an approximate $\bar{Q}_{n j}$ as

$$
\bar{Q}_{n f} \equiv\left(1-V_{n f} R\right)^{-1} V_{n f} \text {. }
$$

Therefore, we ignore the couplings among various $Q_{n f}$ 's. This approximation is similar in spirit to the one introduced in Sec. II.A to decouple $Q_{n}$ from $Q_{m}$. The self-consistent condition now becomes

$$
\langle\bar{T}\rangle=\left\langle\sum_{n} \sum_{f} \bar{Q}_{n j}\right\rangle=0 .
$$

Notice that Eq. (2.27) can be used in two different ways. In the first approach, which is more rigorous, one can assign a self-energy to a subband. In other words,

$$
\sigma_{n f}=|n, f\rangle U_{f}\langle n, f| .
$$

With this particular choice of $\sigma_{n f}$, we have to demand that for each $n, f$,

$$
\left\langle\bar{Q}_{n f}\right\rangle=0 .
$$

There will be $F$ equations to solve for $F$ unknowns, namely $U_{f}$ 's. The index $n$ is immaterial because in CPA all the sites are assumed to be uncorrelated and, therefore, equivalent. Another approach can be adopted whereby we assume an average self-energy for all the subbands, i.e.,

$$
\sigma_{n f}=\mid(n, f\rangle U\langle n, f| .
$$

Thus, we have for each site $n$,

$$
\left\langle\sum_{f} \bar{Q}_{n f}\right\rangle=0 \text {. }
$$

Only one equation is available to solve for one unknown $U$. In either way, the self-consistency is guaranteed because from Eq. (2.7),

$$
\bar{H}_{\text {eff }}-K=\left\langle\sum_{n} \sum_{f} \bar{Q}_{n f}\right\rangle\left(1+R\left\langle\sum_{n} \sum_{f} \bar{Q}_{n f}\right\rangle\right)^{-1}
$$

and, with either Eqs. (2.29) or (2.31), we have

$$
\bar{H}_{\text {eff }}=K \text {. }
$$

We have introduced a single-site and single-band approximation, which is a lower approximation than the single-site approximation by Velicky et $a l .^{16}$ In essence, we adopt an approach which decouples the various selfenergies of subbands so that they can be determined more easily. The decoupling process is done selfconsistently. It should be noticed that the resulting Green's functions for the subbands are still coupled through the Dyson equation. In a separate paper, ${ }^{20}$ the present formalism is applied to the phonon problem in benzene and perdeuterated benzene mixed crystals. It is shown there that Eq. (2.29) leads to the exact solution: (1) When the perturbation is small so that the virtual crystal approximation can be justified; in this case, the off-diagonal elements are identically zero. (2) When
$C_{B}=1, C_{A}=0$ or $C_{A}=1, C_{B}=0$, where $C_{A}$ and $C_{B}$ are concentrations of components $A$ and $B$. Again, the selfenergy is automatically diagonal. On the other hand, it will be shown later that, in the limit of infinite dilution, the present formalism is also reduced to the proper Koster and Slater one-impurity formula, if the bandband couplings are small. It appears that the present formalism would work best for subbands that are sandwiched by the rest of subbands. One might expect that some degree of cancellation could reduce the error introduced by the neglect of off-diagonal elements. Model calculations are being carried out and will be reported later.

It is obvious that the present model would work best when subbands are widely separated. Under this condition, the band-band mixing is minimized. It is, then, justified to ignore the presence of other subbands in dealing with mixed crystals. When the subbands are close to each other, there will be bandband mixing even in the absence of impurities. In this situation, it appears to be more convenient to adopt an alternate viewpoint and associate each subband with index $j$, so that there will be no mixing of bands in the absence of impurities. If we adopt this viewpoint, two types of band-band mixing can be distinguished. In the first place, since $\Delta_{f}$ are, in general, different, there is band-band mixing even in the virtual crystal limit. This is because, contrary to one band only, the transformation that diagonalizes the Hamiltonian of pure crystal $A$ will not, in general, diagonalize that of pure crystal $B$. Alternatively, one can say that $\mathbf{k}$ is a good quantum number in the virtual crystal but $j$ is not. This type of mixing is rigorously accounted for in the present model as reflected from the fact that it yields the exact solution in such a limit. ${ }^{20} \mathrm{~A}$ second type of mixing results from disorder which leads to the mixing of $\mathbf{k}$ states. Although the present model is a lower approximation as compared to the single-site approximation, it does allow for the scrambling of $\mathbf{k}$ not only within each subband but also among subbands. To see this, one would only have to note that, in the limit of infinite dilution, the single impurity level is a function of all the $\mathbf{k}, j$ states in the pure crystal (see Sec. IV). In this point of view, we can say that the present model does not ignore the mixing between subbands caused by impurities, but rather treats it approximately. More discussion on the nature of the approximation will be presented later.

In summary, an approximate $K$ which is selfconsistent and based on a single-site and single-band approximation can now be obtained from Eqs. (2.26) and (2.29) or (2.31). In its applications to phonons and excitons in molecular crystals, different forms of $R$ and $V_{n f}$ are involved. However, the procedure introduced here can be applied in both cases. Because of the simplicity involved, we first apply it to the exciton case and take up phonons in a later section. 


\section{CPA FOR EXCITONS IN DISORDERED MOLECULAR CRYSTALS}

We discuss here molecular crystals with more than one molecule per unit cell. Furthermore, all the sites in the unit cell are assumed to be physically equivalent through the interchange symmetry. ${ }^{21}$ As we mentioned before, the applications of the formulation to be developed in this section include electronic states coupled via spinorbit or vibronic couplings, triplet excitons, and vibrational excitons with Fermi resonance, ${ }^{11}$ or site splittings. ${ }^{12}$

In general, the excitation of a single site in the crystal can be characterized within the tight-binding (or Frenkel exciton ${ }^{22}$ ) formulation by a site excitation energy based on the localized site representation:

$$
\begin{aligned}
\epsilon^{f}=\epsilon_{n \alpha} f & =\left\langle n, \alpha, f\left|H^{0}\right| n, \alpha, f\right\rangle-\left\langle n, \alpha, 0\left|H^{0}\right| n, \alpha, 0\right\rangle \\
& =\Delta \epsilon^{f}+D^{\prime},
\end{aligned}
$$

where $H^{0}$ is the total Hamiltonian of the pure crystal and $f$ and 0 denote the $f$ th excited state and the ground state, respectively; $\Delta \epsilon^{f}$ is the gas phase transition energy and $D^{r}$ accounts for the difference between intermolecular van der Waals interactions in the excited and ground states; $n$ and $\alpha$ refer to the unit cell index and sublattice index, respectively; $f=1,2,3, \cdots$, since we deal with a number of exciton bands which are coupled. When isotopic impurities (or guests) are introduced, it is assumed that the site excitation energy is different from that of the host but not the intersite exciton interactions. We can then define the trap depths as the site excitation energy differences between the guest and the host:

$$
\Delta_{f}=\epsilon_{h}{ }^{f}-\epsilon_{g}{ }^{f},
$$

where $f=1,2,3, \cdots$. Henceforward we denote hosts and guests simply as $A$ and $B$ components. We can, then, write $\Delta_{f}=\epsilon_{B} f-\epsilon_{A}^{f}$. The concentrations of the components in the mixed crystal will be simply $C_{A}$ and $C_{B}$.

The complete mixed crystal Hamiltonian can be displayed in the localized representation $\mathrm{as}^{23}$

$$
\begin{aligned}
& H=\sum_{n \alpha f}|n, \alpha, f\rangle \epsilon_{n, \alpha^{\prime}}\langle n, \alpha, f| \\
& \quad+\sum_{n^{\prime} \alpha^{\prime} f} \sum_{f \neq n \alpha f}|n, \alpha, f\rangle W_{n, \alpha, f, n^{\prime}, \alpha^{\prime}, f^{\prime}}\left\langle n^{\prime}, \alpha^{\prime}, f^{\prime}\right| \\
& \quad=\Delta+H^{0},
\end{aligned}
$$

where $\epsilon_{n, \alpha}{ }^{f}=\epsilon_{A}{ }^{f}$ or $\epsilon_{B}{ }^{f}$, depending on whether the site is occupied by $A$ or by $B . \Delta$ is the perturbation and $W$ is the exciton interaction. It is clear from Eq. (3.3a) that

$$
\Delta=\sum_{n} \sum_{\alpha} \sum_{f}|n, \alpha, f\rangle \Delta_{n \alpha} f\langle n, \alpha, f|,
$$

where

$$
\Delta_{n \alpha}^{f}=\epsilon_{n \alpha^{f}}-\epsilon_{A}^{f} .
$$

To begin with, the pure crystal Hamiltonian $H^{0}$ can be diagonalized in the Bloch representation by virtue of the translational symmetry. The transformation from the localized to the delocalized basis set has the following general form:

$$
|\mathbf{K}, j\rangle=N^{-1 / 2} \sum_{n} \sum_{\alpha} \sum_{f} B_{\alpha, f}(\mathbf{K}, j) \exp \left(i \mathbf{K} \cdot \mathbf{R}_{n \alpha}\right)|n, \alpha, f\rangle
$$

where $\mathbf{R}_{n, \alpha}$ is, of course, the displacement vector from the reference point to the lattice site $n, \alpha$ and $j$ is the branch index. Notice that $B_{\alpha, f}(\mathbf{K}, j)$ cannot be uniquely determined from symmetry alone. Even when bands are decoupled, $B_{\alpha, f}(\mathbf{K}, j)$ cannot be so determined except for some special $\mathbf{k}$ values (such as $\mathbf{k}=0$ ) or under the short-range interaction approximation (the restricted Frenkel limit). ${ }^{24}$ It is obvious from Eq. (3.4) that the pure crystal Green's function can be written as ${ }^{15}$

$$
G^{0}(Z)=(1 / N) \sum_{n, \alpha, f} \sum_{n^{\prime} \alpha^{\prime} f} \sum_{K} \sum_{j}\left\{|n, \alpha, f\rangle B_{\alpha, f}(\mathbf{K}, j) B_{\alpha^{\prime}, f^{\prime}} *(\mathbf{K}, j) \exp \left[i \mathbf{K} \cdot\left(\mathbf{R}_{n \alpha}-\mathbf{R}_{n^{\prime} \alpha^{\prime}}\right)\right]\left\langle n^{\prime}, \alpha^{\prime}, f^{\prime}\right| /[Z-E(\mathbf{K}, j)]\right\}
$$

To deal with the substitutionally disordered system, we first define

$$
R=(Z-K)^{-1} \text {. }
$$

The form of the approximate mixed crystal Hamiltonian $K$ depends on models one chooses to work with. As we have mentioned earlier, in a more rigorous approach [Eq. (2.29)], we can assign a self-energy to an individual band, whereas in our second model we assume an average self-energy for all the bands. It should be noticed that in each case the number of equations which can be used to determine the self-consistent self-energies exactly equals the number of self-energies assumed. Furthermore, self-consistency can be achieved in either case.

In the first approach, we define our approximate Hamiltonian $K$ as

$$
K \equiv H^{0}+\sum_{n} \sum_{\alpha} \sum_{f}|n, \alpha, f\rangle U_{f}\langle n, \alpha, f|
$$

where $U_{f}$ is the self-energy for the subband $f$. We are now in a position to utilize our development in Sec. II. It should be noticed that the presence of more than one molecule per unit cell poses no special complications due to the fact that all molecules in the unit cell are physically equivalent. Referring to Eqs. (2.22), (2.25), (2.28), (3.3), 
and 3.7), we now define $V_{\text {naf }}$ as

$$
V_{n \alpha f} \equiv|n, \alpha, f\rangle\left(\Delta_{n \alpha} f-U_{f}\right)\langle n, \alpha, f|,
$$

and according to Eq. (2.26) we have

$$
\bar{Q}_{n \alpha f} \equiv\left(1-V_{n \alpha f} R\right)^{-1} V_{n \alpha f} .
$$

To assure the self-consistency according to Eq. (2.29), one has to put

$$
\left\langle\bar{Q}_{n \alpha f}\right\rangle=0 \text {. }
$$

According to Eq. (3.3c), $\Delta_{n \alpha}{ }^{f}$ takes on only two values: $\Delta_{n \alpha} f=0$ if site $n \alpha$ is occupied by $A ; \Delta_{n \alpha}{ }^{f}=\epsilon_{B} f-\epsilon_{A} f$ if site $n \alpha$ is occupied by $B$. Therefore, Eq. (3.9) leads to

$$
-C_{A} U_{f} /\left(1+\langle n, \alpha, f|R| n, \alpha, f\rangle U_{f}\right)+C_{B}\left(\epsilon_{B}^{f}-\epsilon_{A}^{f}-U_{f}\right) /\left[1-\langle n, \alpha, f|R| n, \alpha, f\rangle\left(\epsilon_{B}^{f}-\epsilon_{A}^{f}-U_{f}\right)\right]=0 .
$$

Equation (3.10) can be recast in a more familiar form, if we introduce

then

$$
U_{f}^{\prime} \equiv U_{f}-C_{B}\left(\epsilon_{B}^{f}-\epsilon_{A}{ }^{f}\right)=U_{f}-C_{B} \Delta_{f} ;
$$

$$
U_{f}^{\prime}=C_{A} C_{B} \Delta_{f}^{2} /\left[R_{n \alpha f^{-1}}+\left(C_{B}-C_{A}\right) \Delta_{f}+U_{f}^{\prime}\right]
$$

where $R_{n \alpha f} \equiv\langle n, \alpha, f|R| n, \alpha, f\rangle$. Notice that $R_{n \alpha f}$ $(\equiv\langle n, \alpha, f|R| n, \alpha, f\rangle)$ is independent of both $n$ and $\alpha{ }^{25}$ Using Eq. (3.11) again, we can put

$$
U_{f}=C_{B} \Delta_{f}+C_{A} C_{B} \Delta_{f}{ }^{2} /\left(R_{n \alpha f}{ }^{-1}-C_{A} \Delta_{f}+U_{f}\right) .
$$

Equations (3.12) and (3.13) have similar forms as those previously derived by Onodera and Toyozawa ${ }^{6}$ and Velicky et al. ${ }^{16}$ respectively, for the isolated band, except that the trap depth in each case is the trap depth associated with the individual band. ${ }^{26}$

Contrary to the case of one band only, the expression for $R_{n \alpha f}$ is quite complex due to the fact that

$$
R^{-1}=Z-H^{0}-\sum_{n} \sum_{\alpha} \sum_{f}|n, \alpha, f\rangle U_{f}\langle n, \alpha, f|
$$

is no longer diagonal in the $|\mathbf{K}, j\rangle$ representation. From Eq. (3.4), one can conveniently define another set of basis functions $|\mathbf{K}, \alpha, f\rangle$ such that

$$
|\mathbf{K}, j\rangle=\sum_{\alpha} \sum_{f} B_{\alpha f}(\mathbf{K}, j)|\mathbf{K}, \alpha, f\rangle
$$

or

$$
|\mathbf{K}, \alpha, f\rangle \equiv N^{-1 / 2} \sum_{n} \exp \left(i \mathbf{K} \cdot \mathbf{R}_{n \alpha}\right)|n, \alpha, f\rangle .
$$

It is apparent that the self-energy defined in Eq. (3.7) is diagonal in the $|\mathbf{K}, \alpha, f\rangle$ representation:

$\sum_{n^{\prime \prime}} \sum_{\alpha^{\prime \prime}} \sum_{f^{\prime \prime}}\left\langle\mathbf{K}, \alpha, f \mid n^{\prime \prime}, \alpha^{\prime \prime}, f^{\prime \prime}\right\rangle U_{f \prime}^{\prime \prime}\left\langle n^{\prime \prime}, \alpha^{\prime \prime}, f^{\prime \prime} \mid \mathbf{K}^{\prime}, \alpha^{\prime}, f^{\prime}\right\rangle$

$$
=U_{f} \delta_{k k^{\prime}} \delta_{\alpha \alpha^{\prime}} \delta_{f f^{\prime}} .
$$

The pure crystal Green's function in such a representation is given by

$G^{0}(\mathbf{K})_{\alpha f, \alpha^{\prime} f^{\prime}}=\sum_{j}\left\{B_{\alpha f}(\mathbf{K}, j) B_{\alpha^{\prime} f^{\prime}} *(\mathbf{K}, j) /[E-E(\mathbf{K}, j)]\right\}$

which is apparently nondiagonal.

From our definition of the CPA mixed crystal Green's function, we can relate it to the pure crystal
Green's function by virtue of a set of Dyson equations:

$$
\begin{aligned}
R(\mathbf{K})_{\alpha f, \alpha^{\prime} f^{\prime}}=G^{0}(\mathbf{K})_{\alpha f, \alpha^{\prime} f^{\prime}} & +\sum_{\alpha^{\prime \prime}} \sum_{f^{\prime \prime}} G^{0}(\mathbf{K})_{\alpha f, \alpha^{\prime \prime} f^{\prime \prime}} \\
& \times U_{f^{\prime \prime}} R(\mathbf{K})_{\alpha^{\prime \prime} f^{\prime \prime}, \alpha^{\prime} f^{\prime}}
\end{aligned}
$$

$R(\mathbf{K})_{\alpha f, \alpha^{\prime} f^{\prime}}$ can then be solved and substituted in the following equation

$$
R_{n \alpha f}=N^{-1} \sum_{K} R(\mathrm{~K})_{\alpha f, \alpha f}
$$

This equation is a result of the invariance of the trace in unitary transformations. Equations (3.13), (3.18), and (3.19) completely define the mixed crystal Greens' function and self-energies for the interacting bands based on the CPA model. To illustrate how one can utilize these equations, we examine the explicit form of $R_{n \alpha f}$ for simple cases of (1) one degree of freedom only and (2) one molecule per unit cell and two degrees of freedom in the following sections.

In the simple case of one degree of freedom only, we see that Eq. (3.18) is reduced to (we drop the index $f$ ), $R(\mathbf{K})_{\alpha, \alpha^{\prime}}=G^{0}(\mathbf{K})_{\alpha, \alpha^{\prime}}+\sum_{\alpha^{\prime \prime}} G^{0}(\mathbf{K})_{\alpha, \alpha^{\prime \prime}} U R(\mathbf{K})_{\alpha^{\prime \prime}, \alpha^{\prime}}$

Transforming back to the $|\mathbf{K}, j\rangle$ representation,

$$
R(\mathbf{K}, j)=G^{0}(\mathbf{K}, j)+G^{0}(\mathbf{K}, j) U R(\mathbf{K}, j) .
$$

That is

$$
R(\mathbf{K}, j)=[E-E(\mathbf{K}, j)-U]^{-1} .
$$

Therefore,

$$
R(\mathbf{K})_{\alpha, \alpha^{\prime}}=\sum_{j} B_{\alpha}(\mathbf{K}, j) B_{\alpha^{\prime}} *(\mathbf{K}, j) /[E-E(\mathbf{K}, j)-U] .
$$

Equation (3.23a) enables us to calculate $R_{n \alpha}$. According 
to Eq. (3.19),

$$
\begin{aligned}
R_{n \alpha} & =N^{-1} \sum_{K} R(\mathbf{K})_{\alpha, \alpha} \\
& =(N \sigma)^{-1} \sum_{K} \sum_{j}[E-E(\mathbf{K}, j)-U]^{-1},
\end{aligned}
$$

where $\sigma$ is the number of molecules per primitive cell. This is because $\left|B_{\alpha}(\mathbf{K}, j)\right|^{2}=1 / \sigma$ within the restricted Frenkel limit. To convert Eq. (3.23b) to a more familiar form, we first notice that for single band only, we have (see also Ref. 23),

$$
E(\mathbf{K}, j)=\boldsymbol{\epsilon}_{\boldsymbol{A}}+\epsilon(\mathbf{K}, j) .
$$

Furthermore, according to Eq. (3.11),

$$
U=U^{\prime}+C_{B} \Delta .
$$

Substituting $E(\mathbf{k}, j)$ and $U$ into Eq. (3.23b), we obtain

$R_{n \alpha}=(N \sigma)^{-1} \sum_{K} \sum_{j}\left[E-C_{A} \epsilon_{A}-C_{B} \epsilon_{B}-\epsilon(\mathbf{K}, j)-U^{\prime}\right]^{-1}$

and from Eq. (3.12) we have,

$$
U^{\prime}=C_{A} C_{B} \Delta^{2} /\left[R_{n \alpha}{ }^{-1}+\left(C_{B}-C_{A}\right) \Delta+U^{\prime}\right] .
$$

Equations (3.24) completely define $R_{n \alpha}$ and $U^{\prime}$. These equations are identical to those previously derived by Hong and Robinson, ${ }^{7}$ Dubovskii and Konobeev, ${ }^{8}$ and Hoshen and Jortner. ${ }^{9}$

Let's consider now the case where there is only one molecule per unit cell but two degrees of freedom. Equation (3.18) is now reduced to

$$
R(\mathbf{K})_{j, f^{\prime}}=G^{0}(\mathbf{K})_{f, f^{\prime}}+\sum_{f^{\prime \prime}} G^{0}(\mathbf{K})_{f f^{\prime \prime}} U_{f^{\prime \prime}} R(\mathbf{K})_{f^{\prime \prime, f f^{\prime}},}
$$

where $f=1,2$. We have dropped the site index $\alpha$. Equation (3.25) can be solved to give

$$
\begin{aligned}
&\left(\begin{array}{ll}
R(\mathbf{K})_{11} & R(\mathbf{K})_{12} \\
R(\mathbf{K})_{21} & R(\mathbf{K})_{22}
\end{array}\right)=\left[1-G^{0}(\mathbf{K})_{1,1} U_{1}-G^{0}(\mathbf{K})_{2,2} U_{2}+U_{1} U_{2}\left\|G^{0}(\mathbf{K})\right\|\right]^{-1} \\
& \times\left(\begin{array}{cc}
G^{0}(\mathbf{K})_{1,1}-U_{2}\left\|G^{0}(\mathbf{K})\right\| & G^{0}(\mathbf{K})_{1,2} \\
G^{0}(\mathbf{K})_{2,1} & G^{0}(\mathbf{K})_{2,2}-U_{1}\left\|G^{0}(\mathbf{K})\right\|
\end{array}\right),
\end{aligned}
$$

where $\left\|G^{0}(\mathbf{K})\right\|$ is the determinant of the matrix $G^{0}(\mathbf{K})_{f, f^{\prime}}$. The self-energies $U_{1}$ and $U_{2}$ are found from Eqs. (3.13) and (3.19) to be

$$
U_{1}=C_{B} \Delta_{1}+C_{A} C_{B} \Delta_{1}^{2}\left\{\left[N^{-1} \sum_{K} \frac{G^{0}(\mathbf{K})_{1,1}-U_{2}\left\|G^{0}(\mathbf{K})\right\|}{1-G^{0}(\mathbf{K})_{1,1} U_{1}-G^{0}(\mathbf{K})_{2,2} U_{2}+U_{1} U_{2}\left\|G^{0}(\mathbf{K})\right\|}\right]^{-1}-C_{A} \Delta_{1}+U_{1}\right\}^{-1},
$$

with a similar equation for $U_{2}$ by substituting $1 \rightarrow 2$ in Eq. (3.27). $U_{1}$ and $U_{2}$ can thus be obtained by solving the two simultaneous equations. Equations (3.26) and (3.27) are somewhat similar ${ }^{26}$ to the equations derived by Dubovskii and Konobeev ${ }^{8}$ who dealt with isolated bands for the system of crystalline naphthalene with two molecules per unit cell. ${ }^{27}$ However, there are some basic questions as to their assumption that different sites can be averaged independently. We feel that such an approach invariably eliminates some of the guest distributions by imposing an artificial constraint that each sublattice should have guest concentration $C_{g}$. In reality, it is the total concentration (sum of the guests in site 1 and 2) that should be kept constant. This difficulty is absent in our case because we have two degrees of freedom rather than two sites.

We proceed to derive the single parameter CPA for the interacting bands. In this model, we define only one self-energy. In other words, $K$ is assumed to take the following form:

$$
K=H^{0}+\sum_{n} \sum_{\alpha} \sum_{f}|n, \alpha, f\rangle U\langle n, \alpha, f| .
$$

Only one equation can be written down for selfconsistency. According to Eq. (2.31), it is

$$
\left\langle\sum_{f} \bar{Q}_{n \alpha f}\right\rangle=0 \text {. }
$$

From the definition of $V_{n \alpha f}$ in Eq. (3.8a) and the corresponding $\bar{Q}_{n \alpha f}$ in Eq. $(3.8 \mathrm{~b})$, we have [compare to Eq. (3.10)]

$$
\begin{aligned}
& \sum_{f}\left[-C_{A} U /\left(1+R_{n \alpha f} U\right)\right] \\
& +\sum_{f}\left\{C_{B}\left(\epsilon_{B}-\epsilon_{A}-U\right) /\left[1-R_{n \alpha f}\left(\epsilon_{B}-\epsilon_{A}-U\right)\right]\right\}=0 .
\end{aligned}
$$

An expression similar to Eq. (3.11) can now be introduced:

$$
U_{f}^{\prime}=U-C_{B} \Delta_{f}
$$

With the help of Eq. (3.30), Eq. (3.29) can be recast as,

$$
\begin{aligned}
& \sum_{f}\left[1 /\left(1+R_{n \alpha f} U_{f}^{\prime}\right)\right] \\
& \times\left\{U_{f}^{\prime}-C_{A} C_{B} \Delta_{f}^{2} /\left[R_{n \alpha f}^{-1}+\left(C_{B}-C_{A}\right) \Delta_{f}+U_{f}^{\prime}\right]\right\}=0,
\end{aligned}
$$

which is to be compared with Eq. (3.12). 
Because of our definition of $K$ in Eq. (3.28), the mixed crystal Green's function now assumes a very simple form due to the fact that $U$ is diagonal in the $|\mathbf{k}, j\rangle$ representation. Consequently, the Dyson equation is a simple scalar equation in such a representation, namely

$$
R(\mathbf{K}, j)=G^{0}(\mathbf{K}, j)+G^{0}(\mathbf{K}, j) U R(\mathbf{K}, j),
$$

which immediately leads to

$$
R(\mathbf{K}, j)=[E-E(\mathbf{K}, j)-U]^{-1} .
$$

To calculate $R_{n \alpha f}$, we utilize the transformation from delocalized to localized representation $[\mathrm{Eq} .(3.4)]$ and obtain

$$
\begin{aligned}
R_{n \alpha f} & =N^{-1} \sum_{K} \sum_{j}\left|B_{\alpha f}(\mathbf{K}, j)\right|^{2} /[E-E(\mathbf{K}, j)-U] \\
& =N^{-1} \sum_{K} \sum_{j}\left|B_{\alpha f}(\mathbf{K}, j)\right|^{2} /\left[E-E(\mathbf{K}, j)-C_{B} \Delta_{f}-U_{f}^{\prime}\right] .
\end{aligned}
$$

It is convenient to define an auxiliary function $F_{f}(Z)$ such that

where

$$
F_{f}(Z) \equiv \int\left[\rho_{f}(x) d x /(Z-x)\right]
$$

$$
\rho_{f}(E) \equiv N^{-1} \sum_{K} \sum_{j}\left|B_{\alpha f}(\mathbf{K}, j)\right|^{2} \delta[E-E(\mathbf{K}, j)]
$$

can be considered as the projection of the total densityof-states function on the particular degree of freedom $f$. $R_{n a f}$ is, then, related to the auxiliary function $F_{f}(Z)$ through the following expression:

$$
R_{n \alpha f}=F_{f}\left(Z-C_{B} \Delta_{f}-U_{f}^{\prime}\right) .
$$

Similarly, the self-energy is given by

$$
\begin{aligned}
& \sum_{f}\left\{1 /\left[1+U_{f}^{\prime} F_{f}\left(Z-C_{B} \Delta_{f}-U_{f}^{\prime}\right)\right]\right\} \\
& \times\left\{U_{f}^{\prime}-C_{A} C_{B} \Delta_{f}^{2} /\left[F_{f}^{-1}\left(Z-C_{B} \Delta_{f}-U_{f}^{\prime}\right)\right.\right. \\
& \left.\left.+\left(C_{B}-C_{A}\right) \Delta_{f}+U_{f}^{\prime}\right]\right\}=0 .
\end{aligned}
$$

Equations (3.35) and (3.36) are more useful, in practice, than Eqs. (3.13), (3.18), and (3.19). In the former case, only the projections of the total density-ofstates function on each degree of freedom is called for whereas in the latter case, a complete knowledge of the dispersion relation is necessary.

In concluding this section, we would like to investigate the asymptotic behaviors of the self-energies in the limit of infinite dilution. In such a limit, a generalization of Koster and Slater's scheme ${ }^{28}$ leads to the following secular equation for single impurity levels:

where

$$
\left|1-G^{0} \Delta\right|=0 \text {, }
$$

$G_{f f^{\prime}}=N^{-1} \sum_{K} \sum_{j}\left\{B_{\alpha f}(\mathbf{K}, j) B_{\alpha f^{\prime}} *(\mathbf{K}, j) /[E-E(\mathbf{K}, j)]\right\}$

and $\Delta_{f f^{r}}=\Delta_{f} \delta_{f f^{\prime}}$. Equation (3.37) is independent of $\alpha$ as it should be. Equation (3.37) has a form similar to that of the Koster and Slater relation for clusters. ${ }^{29}$

On the other hand, as $C_{B^{-} \rightarrow 0}, C_{A} \rightarrow 1$, Eq. (3.12) becomes,

and

$$
U_{f}^{\prime} \rightarrow C_{B} \Delta_{f}^{2} /\left(R_{n \alpha f^{-1}}-\Delta_{f}\right),
$$

$R_{n \alpha f}=N^{-1} \sum_{K} R(\mathbf{K})_{\alpha f, \alpha f} \rightarrow N^{-1} \sum_{K} G^{0}(\mathbf{K})_{\alpha f, \alpha f}=G_{f j^{0}}$.

The single-impurity levels are, then, given by the poles of $U_{f}^{\prime}$, namely, by solving

$$
G_{f f}^{0}=1 / \Delta_{f}
$$

This equation is the same as Eq. (3.37) if the offdiagonal elements of the Green's function are ignored.

Similarly, in the same limit, Eq. (3.36) is reduced to

$$
\sum_{f}\left\{U_{f}{ }^{\prime}-C_{B} \Delta_{f}{ }^{2} /\left[F_{f}^{-1}(Z)-\Delta_{f}\right]\right\}=0 .
$$

Furthermore, $U \rightarrow U_{f}^{\prime}$, according to Eq. (3.30). Hence,

$$
U \rightarrow(1 / F) \sum_{f}\left\{C_{B} \Delta_{f}^{2} /\left[F_{f}^{-1}(Z)-\Delta_{f}\right]\right\},
$$

where $F$ is the total number of degrees of freedom. ${ }^{25}$ It is, therefore, concluded that, in this model, the single-impurity levels, i.e., the poles of $U$ are simply given by

$$
F_{f}^{-1}(Z)-\Delta_{f}=0
$$

which is equivalent to Eq. $\left(3.37^{\prime \prime \prime}\right)$ because $F_{f}(Z)=G_{f f}{ }^{0}$.

In summary, we see that our Eqs. (3.12) and (3.36) give, in the limit of infinite dilution, single-impurity levels which correspond to the Koster and Slater relation. if the band-band couplings are neglected. As we mentioned earlier, this is due to the fact that we have employed an approximate $Q_{n f}$ defined in Eq. (2.26), rather than the more rigorous $\mathrm{Eq}$. (2.19). The entire formulation presented here is not the simple single-site approximation but rather the so-called "single-site and single-band approximation". Within this approximation, the best available self-energy is the selfconsistent self-energy, i.e., the one based on the Coherent Potential Approximation.

\section{CPA FOR PHONONS IN DISORDERED MOLECULAR CRYSTALS}

Phonons in substitutionally disordered systems have been treated before. In fact, the earlier works on disordered systems concerned mostly the frequency distributions of disordered lattices. Historically, the frequency distribution of a disordered chain was first treated by Dyson. ${ }^{30}$ The moment trace method of Domb et al. ${ }^{31}$ was also developed for phonons. The Negative Factor Counting (NFC) method by Dean and his coworkers ${ }^{32}$ dealt with lattice vibrations as did the Green's function method and CPA introduced by Taylor. ${ }^{5}$ 
Despite the abundance of literature on phonons in disordered solids, little has been done on the lattice dynamics of disordered molecular crystals. Even for the ordered systems, i.e., pure molecular crystals, theoretical calculations, and experimental data $^{33}$ on lattice dynamics have just begun to emerge. For "atomic" crystals, substitutional disorder frequently means a simple mass defect. The disordered systems can, then, be characterized by a single parameter, namely, the mass ratio between the host and the guest. In particular, Taylor's CPA for phonons is only suitable for cubic atomic crystals and really deals with multiple bands which are degenerate and noninteracting. For molecular crystals, we can make the assumption that the molecules are rigid and thus decouple the intermolecular vibrations from the intramolecular vibrations. However, we have to deal with not only translations but also rotations. The corresponding disordered systems can be viewed as interacting multiple bands, with each subband characterized by a disorder strength which is either the mass ratio or the moment of inertia ratio between the host and the guest. Substitional disorder in this case means that (1) guests can be introduced into the host lattice substitutionally and (2) no force constant changes are involved. Such theoretical simplifications are met very closely by the systems of isotopic mixed crystals, such as benzene- $h_{6}$ and benzene- $d_{6}$.

In the following development, we again deal with molecular crystals which may have more than one molecule per unit cell. The various sites in the lattice are assumed to be physically equivalent through interchange symmetry. ${ }^{21}$ Within the harmonic approximation, the mixed crystal Hamiltonian can be written as

$$
\begin{aligned}
& H=\frac{1}{2} \sum_{n} \sum_{\alpha} \sum_{f} \xi_{\alpha, f^{2}}(n, t) / \eta_{\alpha, f}(n) \\
& +\frac{1}{2} \sum_{n} \sum_{\alpha} \sum_{f} \sum_{n^{\prime}} \sum_{\alpha^{\prime}} \sum_{f^{\prime}} u_{\alpha, f}(n, t) \\
& \quad \quad \times \Phi\left(n, \alpha, f, n^{\prime}, \alpha^{\prime}, f^{\prime}\right) u_{\alpha^{\prime}, f^{\prime}}\left(n^{\prime}, t\right),
\end{aligned}
$$

where $n$ and $\alpha$ are unit cell and sublattice indices, respectively; $f$ denotes th six degrees of freedom ( 3 translations and 3 rotations); $\xi$ is either the linear or angular momentum; $\eta$ is either the mass or the moment of inertia and $t$ is time. It is apparent that

and

$$
\xi_{\alpha, f}(n, t)=\eta_{\alpha, f}(n) \dot{u}_{\alpha, f}(n, t)
$$

$\Phi\left(n, \alpha, f, n^{\prime}, \alpha^{\prime}, f^{\prime}\right)=\partial^{2} V / \partial u_{\alpha, f}(n) \partial u_{\alpha^{\prime}, f^{\prime}}\left(n^{\prime}\right)$,

where $V$ is the potential energy of the crystal.

It is more convenient in dealing with phonons to use the time independent equation of motion. ${ }^{34}$ The equation of motion for mixed crystals can be wirtten in matrix form as ${ }^{34}$

$$
\sum_{n^{\prime}} \sum_{\alpha^{\prime}} \sum_{f^{\prime}} L\left(n, \alpha, f, n^{\prime}, \alpha^{\prime}, f^{\prime} ; \omega^{2}\right) u_{\alpha^{\prime}, f^{\prime}}\left(n^{\prime}\right)=0,
$$

where

$$
\begin{aligned}
L\left(n, \alpha, f, n^{\prime}, \alpha^{\prime}, f^{\prime} ; \omega^{2}\right)= & \eta_{\alpha, f}(n) \omega^{2} \delta_{n n^{\prime}} \delta_{\alpha \alpha^{\prime}} \delta_{f f^{\prime}} \\
& -\Phi\left(n, \alpha, f, n^{\prime}, \alpha^{\prime}, f^{\prime}\right) .
\end{aligned}
$$

$\eta_{\alpha f}(n)$ takes on either $\eta_{A}{ }^{f}$ or $\eta_{B}{ }^{f}$, depending on whether the site $n, \alpha$ is occupied by $A$ or $B . \omega$ is the frequency.

We now define the Green's function $a^{34}$

$$
\mathbf{G}=\mathrm{L}^{-1} \text {, }
$$

which is equivalent to the one used by Taylor. ${ }^{5}$

The relation between the Green's functions for perturbed and unperturbed crystals can be obtained from corresponding equations of motion. If we define $\mathbf{L}^{0}$ as the pure crystal analog of $\mathbf{L}$, we find that ${ }^{35}$

$$
\mathbf{L}=\mathbf{L}^{0}-\delta \mathbf{L}
$$

and

$\delta L\left(n, \alpha, f, n^{\prime}, \alpha^{\prime}, f^{\prime} ; \omega^{2}\right)=\eta_{A}{ }^{f} \Delta_{\alpha f}(n) \omega^{2} \delta_{n n^{\prime}} \delta_{\alpha \alpha^{\prime}} \delta_{f f^{\prime}}$,

where

$$
\Delta_{\alpha f}(n)=\left[\eta_{A^{f}}-\eta_{\alpha f}(n)\right] / \eta_{A^{f}}
$$

is the random parameter. Notice that $\Delta_{\alpha f}(n)=0$ if the site $n, \alpha$ is occupied by the host $(A)$ and $\Delta_{\alpha f}(n)=$ $\left(\eta_{A}^{f}-\eta_{B} f\right) / \eta_{A}{ }^{f} \equiv \Delta^{f}$ if it is occupied by the guest $(B)$.

From Eqs. (4.4) and (4.5a), we obtain the usual Dyson equation

where

$$
\mathrm{G}=\mathrm{G}^{0}+\mathrm{G}^{0} \delta \mathrm{LG}
$$

$$
\begin{aligned}
& G_{n \alpha f, n^{\prime} \alpha^{\prime} f^{\prime}}=\frac{1}{N\left(\eta_{A}^{f} \eta_{A^{f}}^{f^{\prime}}\right)^{1 / 2}} \\
& \times \sum_{j} \sum_{K} \frac{B_{\alpha f}(\mathbf{K}, j) B_{\alpha^{\prime} j^{\prime}} *(\mathbf{K}, j) \exp \left[i \mathbf{K} \cdot\left(\mathbf{R}_{n \alpha}-\mathbf{R}_{n^{\prime} \alpha^{\prime}}\right)\right]}{\omega^{2}-\omega^{2}(\mathbf{K}, j)}
\end{aligned}
$$

is the pure crystal Green's function.

Following the development in Sec. II, we proceed to define an approximate average Green's function as

$$
\mathbf{R}=\mathbf{G}^{\mathbf{0}}+\mathbf{G}^{\mathbf{0}} \mathbf{\Sigma} \mathbf{R} \text {. }
$$

It follows immediately from Eqs. (4.6) and (4.8) that

$$
\mathbf{G}=\mathbf{R}+\mathbf{R}(\boldsymbol{\delta} \mathbf{L}-\mathbf{\Sigma}) \mathbf{G} \text {. }
$$

Equation (4.9) is equivalent to Eq. (2.9); the former is for phonons whereas the latter is for excitons. Furthermore, we can establish the following equivalence between excitons and phonons:

$$
\begin{gathered}
\sum_{n} \sum_{\alpha} \sum_{f} V_{n \alpha f} \Rightarrow \delta L^{\mathrm{op}}-\mathbf{\Sigma}^{\mathrm{op}} . \\
\text { (exciton) (phonon) }
\end{gathered}
$$

To be more precise, we have used the superscript op to denote the corresponding operators. It is worth noting that, similar to the exciton case, $\delta L^{o p}$ is always diagonal in the localized representation whereas $\Sigma^{\circ p}$ is, in 
general, nondiagonal. However, within our single-site single-band approximation, it is consistent to assume that $\boldsymbol{\Sigma}$ is diagonal. This assumption enables us to write down the self-consistent expressions in a straightforward algebraic form rather than the cumbersome matrix notation.

We first assume that the matrix elements of $\boldsymbol{\Sigma}$ take the following form,

$$
\Sigma\left(n, \alpha, f, n^{\prime}, \alpha^{\prime}, f^{\prime}\right)=\Sigma_{j} \delta_{n n^{\prime}} \delta_{\alpha \alpha^{\prime}} \delta_{f f^{\prime}} .
$$

Using our Eqs. (3.8b) and (3.9), we have, for phonons:

$-C_{A} \Sigma_{f} /\left(1+\Sigma_{f} R_{n \alpha j}\right)$

$+C_{B}\left(\eta_{A}^{f} \Delta^{f} \omega^{2}-\Sigma_{f}\right) /\left[1-\left(\eta_{A}^{f} \Delta^{f} \omega^{2}-\Sigma_{f}\right) R_{n \alpha f}\right]=0$,

where $R_{n \alpha f}=R\left(n, \alpha, f, n, \alpha, f ; \omega^{2}\right)$; Eq. (4.12) is analogous to Eq. (3.10).

Equation (4.12) can be recast in a more familiar form:

$$
\Sigma_{f}-C_{B} \eta_{A} \Delta^{f} \omega^{2}-\Sigma_{f}\left(\eta_{A}^{f} \Delta^{f} \omega^{2}-\Sigma_{f}\right) R_{n \alpha f}=0
$$

which is similar in form to the one previously derived by Taylor ${ }^{5}$ for atomic crystals.

To evaluate $R$ from Eq. (4.8), we again use the transformation

$$
u(\mathbf{K}, \alpha, f)=N^{-1 / 2} \sum_{n} \exp \left(i \mathbf{K} \cdot \mathbf{R}_{n \alpha}\right) u_{\alpha, f}(n) .
$$

'The pure crystal Green's function in such a representation is given by

$$
\begin{aligned}
& G^{0}(\mathbf{K})_{\alpha f, \alpha^{\prime} f^{\prime}}=\left(\eta_{A}^{f} \eta_{A^{\prime}}\right)^{\prime-1 / 2} \\
& \quad \times \sum_{j} B_{\alpha f}(\mathbf{K}, j) B_{\alpha^{\prime} f^{\prime}} *(\mathbf{K}, j) /\left[\omega^{2}-\omega^{2}(\mathbf{K}, j)\right],
\end{aligned}
$$

which is the phonon analog of Eq. (3.17). As in Eqs. (3.18) and (3.19), we can write down

$$
\begin{aligned}
& R(\mathbf{K})_{\alpha f, \alpha^{\prime} f^{\prime}}=G^{0}(\mathbf{K})_{\alpha f, \alpha^{\prime} f^{\prime}} \\
& \quad+\sum_{\alpha^{\prime \prime}} \sum_{f^{\prime \prime}} G^{0}(\mathbf{K})_{\alpha f, \alpha^{\prime \prime} f^{\prime \prime}} \Sigma_{f^{\prime \prime}} R(\mathbf{K})_{\alpha^{\prime \prime} f^{\prime \prime}, \alpha^{\prime} f^{\prime}}
\end{aligned}
$$

and

$$
R_{n \alpha f}=N^{-1} \sum_{K} R(\mathbf{K})_{\alpha f, \alpha f} .
$$

The CPA self-energy is, thus, completely defined by Eqs. (4.13), (4.15), (4.16), and (4.17). Equation (4.13) can also be recast as

$\Sigma_{f}^{\prime}=C_{A} C_{B}\left(\eta_{A}{ }^{f} \Delta^{f} \omega^{2}\right)^{2} /\left[R_{n \alpha f}{ }^{-1}+\left(C_{B}-C_{A}\right)\left(\eta_{A}{ }^{f} \Delta^{f} \omega^{2}\right)+\Sigma_{f}{ }^{\prime}\right]$,

where

$$
\Sigma_{f}^{\prime} \equiv \Sigma_{f}-C_{B}\left(\eta_{A}^{f} \Delta^{f} \omega^{2}\right) .
$$

The resemblance between disordered excitons and phonons now becomes clear: the degree of disorder is measured by the trap depth for excitons and it is measured by the parameter $\eta_{A}^{f} \Delta^{f} \omega^{2}$ for phonons. The latter parameter is related to the percentage mass or moment of inertia difference.
To use the second approach, we should notice that $\bar{Q}_{n \alpha f}$ has the same dimensions as $V_{n \alpha f}$. In our problem, both rotations and translations are involved. To ensure that all the $\bar{Q}_{n \alpha f}$ 's have the same dimensions, we would have to use the transformation:

$$
v_{\alpha, f}(n, t)=\left(\eta_{A}^{f}\right)^{1 / 2} u_{\alpha, f}(n, t) .
$$

It can be easily shown that in the $v_{\alpha f}(n, t)$ representation

and

In other words,

$$
\begin{aligned}
& V_{n \alpha f^{\prime}}=V_{n \alpha f} / \eta_{A}{ }^{f} \\
& R_{n \alpha f^{\prime}}=\eta_{A}{ }^{\prime} R_{n \alpha f} .
\end{aligned}
$$

$$
\bar{Q}_{n \alpha f}{ }^{\prime}=\bar{Q}_{n \alpha f} / \eta_{A}{ }^{f}
$$

The same algebra presented in Sec. II can be carried out for the primed system. This would lead to

$$
\sum_{f}\left\langle\bar{Q}_{n \alpha f^{\prime}}\right\rangle=0
$$

which is the same as

$$
\sum_{f}\left\langle\bar{Q}_{n \alpha f} / \eta_{A}{ }^{f}\right\rangle=0 .
$$

Similar to Eq. (3.31), we now have, for phonons:

$\sum_{f}\left[\eta_{A}{ }^{f}\left(1+R_{n \alpha f} \Sigma_{f}^{\prime}\right)\right]^{-1}$

$\times\left\{\Sigma_{f}^{\prime}-\frac{C_{A} C_{B}\left(\eta_{A}{ }^{f} \Delta^{f} \omega^{2}\right)^{2}}{R_{n \alpha f}{ }^{-1}+\left(C_{B}-C_{A}\right)\left(\eta_{A}^{f} \Delta^{f} \omega^{2}\right)+\Sigma_{f}^{\prime}}\right\}=0$.

where $\Sigma_{f}^{\prime}=\eta_{A}{ }^{f} \Sigma-C_{B} \eta_{A}{ }^{f} \Delta^{f} \omega^{2}$, where $\eta_{A}^{f} \Sigma$ is the selfenergy in the unprimed system.

$R_{n \alpha f}$ can be written easily as

$R_{n \alpha f}=\left(1 / N \eta_{A}^{f}\right) \sum_{K} \sum_{j}\left|B_{\alpha f}(\mathbf{K}, j)\right|^{2} /\left[\omega^{2}-\omega^{2}(\mathbf{k}, j)-\Sigma\right]$.

An auxiliary function can now be defined as

$$
F_{j}\left(Z^{2}\right) \equiv\left(1 / \eta_{A} f\right) \int \nu_{f}(y) d y /\left(Z^{2}-y^{2}\right),
$$

where

$$
\nu_{f}(\omega) \equiv N^{-1} \sum_{K} \sum_{j}\left|B_{\alpha f}(\mathbf{K}, j)\right|^{2} \delta[\omega-\omega(\mathbf{K}, j)]
$$

is the projection of the total density-of-states function onto the particular degree of freedom $f$. We find that

$$
R_{n \alpha f}=F_{f}\left(Z^{2}-\Sigma\right) \text {. }
$$

Consequently,

$$
\sum_{f}\left[\eta_{A}{ }^{f}+\Sigma_{f}^{\prime} F_{f}\left(Z^{2}-\Sigma\right) \eta_{A^{\prime}}\right]^{-1}
$$

$$
\times\left\{\Sigma_{f}^{\prime}-\frac{C_{A} C_{B}\left(\eta_{A}{ }^{f} \Delta^{f} \omega^{2}\right)^{2}}{F_{f}^{-1}\left(Z^{2}-\Sigma\right)+\left(C_{B}-C_{A}\right) \eta_{A}{ }^{f} \Delta^{\prime} \omega^{2}+\Sigma_{f}^{\prime}}\right\}=0
$$

which is, of course, analogous to Eq. (3.36).

Finally, it is easy to see that when $C_{B} \rightarrow 0 ; C_{A} \rightarrow 1$, 
Eq. (4.18) is reduced to

$$
\Sigma_{f}^{\prime} \rightarrow C_{B}\left(\eta_{A}^{f} \Delta^{f} \omega^{2}\right)^{2} /\left(R_{\left.n \alpha f^{-1}-\eta_{A}{ }^{f} \Delta^{f} \omega^{2}\right),}\right.
$$

and

$R_{n \alpha f}=N^{-1} \sum_{K} R(\mathbf{K})_{\alpha f, \alpha f} \rightarrow N^{-1} \sum_{K} G^{0}(\mathbf{K})_{\alpha f, \alpha f} \equiv G_{f f^{0}}$.

Similarly, Eq. (4.24) is reduced to

$\Sigma \rightarrow(1 / F) \sum_{f} C_{B}\left(\eta_{A}^{f} \Delta^{f} \omega^{2}\right)^{2} / \eta_{A}^{f}\left[F_{f}^{-1}\left(Z^{2}\right)-\eta_{A}^{f} \Delta^{f} \omega^{2}\right]$

The poles of $\Sigma_{f}$ and $\Sigma$ are given by

$$
G_{f f}^{0} \eta_{A}{ }^{f} \Delta^{f} \omega^{2}=1,
$$

and

$$
F_{f}\left(Z^{2}\right) \eta_{A}^{f} \Delta^{f} \omega^{2}=1,
$$

which is equivalent to

$$
\left(\Delta^{j} \omega^{2} / N\right) \sum_{K} \sum_{j}\left|B_{\alpha f}(\mathbf{K}, j)\right|^{2} /\left[\omega^{2}-\omega^{2}(\mathbf{K}, j)\right]=1,
$$

the well-known equation for single-impurity levels for phonons ${ }^{36}$ when the band-band couplings are small.

It has been demonstrated that mixed crystal studies can be utilized to elucidate the intermolecular interactions in the pure crystal. Various investigations in the past have been successful to varying degrees in leading towards a better understanding of exciton density-ofstates-functions, pairwise interactions and the complete dispersion relations of an isolated exciton band. Bernstein and Robinson ${ }^{37}$ and Kopelman ${ }^{38}$ have studied the infrared spectra of isotopic mixed crystals of benzene and obtained pairwise interactions for vibrational excitons. In the case of the ${ }^{1} B_{2 u}$ naphthalene exciton band, Hanson et al. ${ }^{39}$ have utilized the monomer data to confirm the validity of a density-of-statesfunction determined independently from the hot band experiments. ${ }^{40}$ Hong and Robinson ${ }^{7}$ studied the heavily doped mixed crystals of naphthalene and were able to establish the reliability of the same density-of-states function. Lately, Hanson ${ }^{41}$ and Hong and Kopelman ${ }^{42}$ analyzed the resonance pair spectra of ${ }^{1} B_{2 u}$ naphthalene exciton band and acquired the complete exciton dispersion relation for the particular band, In a separate paper by Hong and Kopelman, ${ }^{43}$ it was also demonstrated how the heavily doped mixed crystal data can be used in either determining or confirming a particular exciton dispersion relation. All this work was done for an "isolated" band and its success bore witness to the fruitfulness of mixed crystal studies.

For the interacting bands, experimental or theoretical studies are scarce. Recently, Prasad and Kopelman ${ }^{44}$ studied the intra- and intermolecular vibrational modes of naphthalene in Raman spectra. However, for the particular molecular crystal, it appeared that the restricted Frenkel exciton concept was useful in describing the energy band of a so-called "rotational exciton".
Therefore, much of the discussion could be based on a noninteracting band picture. However, a parallel study by Hong and Kopelman ${ }^{20}$ on Raman spectra of isotopic mixed crystals of benzene indicated that the interacting band picture was required. The various Davydov components of lattice vibrations are entwined in crystalline benzene. ${ }^{45}$ The development presented here is, therefore, geared to deal with such a complicated situation. It should be emphasized that in the interacting band picture, the mixed crystal studies can be utilized to study the couplings between the bands, either through Eqs. (4.13), (4.16), and (4.17) or through (4.20) and (4.21) [or (4.23) and (4.24)]. In particular, for the latter model, a particular projection of the total density-of-state function onto various degrees of freedom can be critically examined by comparing the theoretical predictions with spectral data. Thus, a mixed crystal study would shed some light on the validity of a particular partition or projection of the density-of-state function onto various degrees of freedom which, in turn, would yield some information concerning the band-band interactions. Some applications of this paper to the Raman spectra of heavily doped mixed crystals of benzene and pertinent experimental data have been presented in a separate publication. $^{20}$

\section{CONCLUSION}

We have developed a CPA theory for excitons and phonons in substitutionally disordered molecular crystals with interacting bands. The assumptions used here are: (1) The guests are introduced into the host lattice substitutionally and (2) no perturbations on either the exciton interactions or force constants are involved. Within these assumptions and the single-site single-band approximation, the self-energies of perturbed crystals can be easily determined by imposing the self-consistent conditions. In particular, two approximations were introduced. In one approximation the self-energies were assumed to be site-independent but dependent on the various degrees of freedom. In the other approximation, the self-energy is independent of both site and degrees of freedom. Because of the selfconsistency, these self-energies are the best available self-energies within the single-site single-band approximation. Interacting bands are quite common, especially for phonons. The development presented here should have wide applications in dealing with electronic and vibrational excitons and lattice phonons in disordered solids.

* Supported by NSF Grant GP-18718 and NIH Grant NS08116. † Present address: Department of Chemistry, State University of New York at Stony Brook, Stony Brook, NY 11790.

1 For general review and bibliographies see N. F. Mott, Advan. Phys. 16, $49(1967) ;$ N. F. Mott and W. D. Twose, ibid., 10, 107 (1961); J. Hori, Spectral Properties of Disordered Chains and Lattices (Pergamon, Oxford, England, 1968); A. A. Maradudin, E. W. Montroll, G. H. Weiss, and I. P. Ipatova, Solid State Physics Suppl. 3, 353 (1971). 
${ }^{2}$ M. Lax, Rev. Mod. Phys. 23, 287 (1951); Phys. Rev. 85, 621 (1952).

${ }^{3} \mathrm{~F}$. Yonezawa and T. Matsubara, Progr. Theoret. Phys. (Kyoto) 31, 357 (1964); 35, 357 (1966); 35, 759 (1966); 37, $1346(1967)$.

4 P. Soven, Phys. Rev. 156, 809 (1967); 178, 1136 (1969).

${ }^{5}$ D. W. Taylor, Phys. Rev. 156, 1017 (1967).

${ }^{6}$ Y. Onodera and Y. Toyozawa, J. Phys. Soc. Japan 24, 341 (1968).

${ }^{7}$ H. K. Hong and G. W. Robinson, J. Chem. Phys. 52, 825 (1970); 54, 1369 (1971).

8 O. A. Dubovskii and Yu. V. Konobeev, Fiz. Tverd. Tela 12, 405 (1970), [Sov. Phys. Solid State 12, 321 (1970)]

${ }^{9} \mathrm{~J}$. Hoshen and J. Jortner, Chem. Phys. Lett. 5, 351 (1970).

${ }^{10}$ See, for example, W. Cochran and G. S. Pawley, Proc. Roy. Soc. A 280, 1 (1964); G. S. Pawley, Phys. Stat. Sol. 20, 347 (1967); G. S. Pawley and S. J. Cyvin, J. Chem. Phys. 52, 4073 (1970) ; G. S. Pawley and E. A. Yeats, Solid State Commun. 7, 385 (1969).

11 R. Kopelman, J. Chem. Phys, 44, 3547 (1966); E. R. Bernstein, S. D. Colson, R. Kopelman, and G. W. Robinson, J. Chem. Phys. 47, 5596 (1968). See also Ref. 12.

${ }_{12}$ E. R. Bernstein, S. D. Colson, R. Kopelman, and G. W. Robinson, J. Chem. Phys. 48, 5596 (1968) ; E. R. Bernstein, J. Chem. Phys. 50, 4842 (1969) ; E. R. Bernstein and G. W. Robinson, J. Chem. Phys. 49, 4962 (1968); E. R. Bernstein, S. D. Colson, D.S. Tinti, and G. W. Robinson, J. Chem. Phys. 48, 4632 (1968); R. Kopelman, J. Chem. Phys. 47, 3227 (1967).

${ }^{13}$ C. A. Hutchison Jr., and B. W. Mangum, J. Chem. Phys. 29, 952 (1958) ; 32, 1261 (1958); 34, 908 (1961).

${ }_{14}$ G. C. Nieman and G. W. Robinson, J. Chem. Phys. 39, 1298 (1963); 37, 2150 (1962); H. Sternlicht, G. C. Nieman, and G. W. Robinson, J. Chem. Phys. 39, 1610 (1963); J. L. Katz, J. Jortner, S.-I. Choi, and S. A. Rice, J. Chem. Phys. 39, 1897 (1963).

${ }^{15}$ B. S. Sommer and J. Jortner, J. Chem. Phys. 50, 187, 839 (1969).

${ }_{16}$ B. Velicky, S. Kirkpatrick, and H. Ehrenreich, Phys. Rev. 175, 747 (1968) ; S. Kirkpatrick, B. Velicky, and H. Ehrenreich, Phys. Rev. B1, 3250 (1970).

${ }_{17}$ See Ref. 16 and also J. Hoshen and J. Jortner, J. Chem, Phys. 56, 933 (1972).

18 M. V. Klein, Phys. Rev. 131, 1500 (1963).

19 P. N. Sen and M. H. Cohen, A morphous and Liquid Semiconductors, edited by M. H. Cohen and G. Lucovsky (NorthHolland, Amsterdam, 1972), p. 147.

${ }^{20} \mathrm{H}$. K. Hong and R. Kopelman, J. Chem. Phys. 58, 384 (1973).

21 R. Kopelman, J. Chem. Phys. 47, 2631 (1967).

${ }^{22}$ J. Frenkel, Phys. Rev. 37, 17, 1276 (1931); A. S. Davydov, Theory of Molecular Excitons (McGraw-Hill, New York, 1962); Usp. Fiz. Nauk 82, 393 (1964) [Sov. Phys. Usp. 7, 145 (1964)]; A. S. Davydov, Theory of Molecular Excitons (Plenum, New York, 1971).

${ }^{23}$ Notice that we have included the diagonal elements in $\mathbf{H}^{0}$. For an isolated band, it is usually possible to write the mixed crys tal Hamiltonian as (see Ref. 16) $\mathbf{H}=\mathrm{D}+\mathrm{W}$, where $\mathrm{D}$ contains only the diagonal elements and $W$ contains only the offdiagonal elements. Furthermore, the pure crystal Hamiltonians of both $A$ and $B\left(\mathbf{H}_{A}{ }^{0}\right.$ and $\left.\mathbf{H}_{B}{ }^{0}\right)$ contain $W: H_{A}{ }^{0}=\epsilon_{A} l+W$, and $\mathrm{H}_{B}{ }^{0}=\epsilon_{B} \mathbf{1}+\mathrm{W}$. Thus, it is possible to diagonalize both $\mathrm{H}_{A}{ }^{0}$ and $\mathbf{H}_{B}{ }^{0}$ with the same set of $B_{\alpha}(\mathbf{k}, j)$.

This is not true for the case of interacting bands. Now, $\mathrm{H}_{A}{ }^{0}=$ $\varepsilon_{A}+W, H_{B}{ }^{0}=\varepsilon_{B}+W$. Although $\varepsilon_{A}$ and $\varepsilon_{B}$ still contain only diagonal elements, they are no longer simple constant multiples of the unit matrix. The $B_{\alpha f}(\mathbf{k}, j)$ 's are different for components $A$ and $B$. In other words, the density-of-states functions for $A$ and $B$ are not congruent to each other. Under these circumstances, it is more convenient to decompose the mixed crystal Hamiltonian as we do in $\mathbf{E q}$. (3.3a). The $\boldsymbol{B}_{\alpha f}(\mathbf{k}, j)$ used throughout later sections is understood to be associated with the host $(A)$ and not the guest $(B)$. The same thing is true for phonons discussed in Sec. IV.

${ }^{24}$ S. D. Colson, R. Kopelman, and G. W. Robinson, J. Chem. Phys. 47, 27, 5462 (1967) ; G. W. Robinson, Ann. Rev. Phys. Chem. 31, $429(1970)$.

${ }^{26}$ Here, we have put $(n, \alpha, f|R| n, \alpha, f)=R_{n \alpha f}$. It should be pointed out that $R_{n \alpha f}$ is independent of $n, \alpha$. We can define $R_{f} \equiv R_{n \alpha f}$. Thus, when we take the trace of the Green's function, we have TraceG $=\Sigma_{n} \Sigma_{\alpha} \Sigma_{f}\langle n, \alpha, f|R| n, \alpha, f\rangle=N_{\sigma} \Sigma_{f} R_{f}$, where $\sigma$ is the total number of molecules per primitive cell. Similarly, the over-all density-of-states function $g(E)$ is given by $g(E)=(1 / N \sigma F) I m$ TraceG $=(1 / F) \Sigma_{f} I m R_{f}$, where $F$ is the total number of degrees of freedom.

${ }_{26}$ There appears to be some confusion with regard to different definitions of self-energies by different authors, particularly those defined in Refs. 6,16 , and the present paper. Direct comparison is impossible because we are dealing with multibands and, as explained in Ref. 23, we have to absorb the diagonal terms into $H^{0}$ also. However, in the limit of one band only our definitions of self-energies are related to those of other authors by $U_{f}=\Sigma_{1}-\epsilon_{A}$,

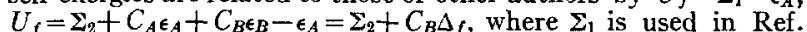
$16 ; \Sigma_{2}$ is used in Ref. 6 . In other words, from Eq. (3.11), we have also $U_{f}^{\prime}=\Sigma_{2}$. Care must be taken in comparing our results with those in Ref. 16. For example, using the above relations, Eq. (3.13) is found to be equivalent to $\Sigma_{1}=C_{A} \epsilon_{A}+C_{B \epsilon_{B}}+$ $C_{A} C_{B} \Delta f^{2} /\left(R_{n \alpha f^{-1}}-C_{A} \epsilon_{B}-C_{B} \epsilon_{A}+\Sigma_{1}\right)$, which is given in Ref. 16 as $\Sigma_{1}=\epsilon+C_{A} C_{B} \Delta_{f}^{2} /\left(R_{n a f}-1+\epsilon+\Sigma_{1}\right)$, because by their definition, $\epsilon_{A}=-\epsilon_{B}$ and so $-C_{A} \epsilon_{B}-C_{B} \epsilon_{A}=C_{A \epsilon_{A}}+C_{B} \epsilon_{B}=\epsilon$.

27 Their formulation is more general. In principle, it can be applied to cases where no interchange symmetry exists. However, as we point out in the text, their assumptions are questionable.

${ }^{28}$ G. F. Koster and J. C. Slater, Phys. Rev. 95, 1167 (1954); 94, 1208 (1954) ; G. F. Koster, Phys. Rev. 95, 1436 (1954).

29 H. K. Hong and R. Kopelman, J. Chem. Phys. 55, 724 (1971); ibid. 57, 3888 (1972).

${ }^{30}$ F. J. Dyson, Phys. Rev. 92, 1331 (1953).

${ }^{31}$ C. Domb, A. A. Maradudin, E. W. Montroll, and G. H. Weiss, Phys. Rev. 115, 18, 24 (1959).

${ }_{32}$ P. Dean, Proc. Phys. Soc. (London), 73, 4136 (1959); Proc. Roy. Soc. (London) A254, 507 (1960); A260, 2636 (1961); P. Dean and J. L. Martin, ibid. A259, 409 (1960); P. Dean and M. D. Bacon, ibid. A283, 64 (1965).

${ }^{33}$ For some theoretical calculations see Ref. 10. Experimental work on molecular crystals using inelastic neutron scattering were reported by P. A. Reynolds, J. K. K jems, and J. W. White, J. Chem. Phys. 56, 2928 (1972), G. Dolling and B. M. Powell, Proc. Roy. Soc. (London) 319, 209 (1970), and others.

${ }^{34}$ For example, see A. A. Maradudin, E. W. Montroll, G. H. Weiss, and I. P. Ipatova, Solid State Phys. Suppl. 3, 65 (1971). ${ }^{35}$ See Ref. 34, p. 361.

${ }^{36}$ For example, see Ref. 34, p. 459.

37 E. B. Bernstein and G. W. Robinson, J. Chem. Phys. 49, 4962 (1968).

${ }_{38}$ R. Kopelman, J. Chem. Phys. 47, 3227 (1967).

${ }^{38}$ D. M. Hanson, R. Kopelman, and G. W. Robinson, J. Chem. Phys. 51, $212(1969)$.

${ }_{40}$ S. D. Colson, D. M. Hanson, R. Kopelman, and G. W. Robinson, J. Chem. Phys. 48, 2215 (1968).

41 D. M. Hanson, J. Chem. Phys. 52, 3409 (1970).

42 H. K. Hong and R. Kopelman, Phys. Rev. Lett. 25, 1030 (1970); J. Chem. Phys. 55, 724 (1971).

${ }_{43} \mathrm{H}$. K. Hong and R. Kopelman, J. Chem. Phys. 55, 5380 (1971).

4 P. N. Prasad and R. Kopelman, J. Chem. Phys. 57, 863 (1972).

${ }_{45}$ See, for example, calculations by D. A. Oliver and $S$. $H$. Walmsley, Mol. Phys. 17, 617 (1969); also assignments by M. Ito and T. Shigeoka, Spectrochim. Acta 22, 1029 (1966). 